OPEN ACCESS

Edited by:

T. Alexander Quinn,

Dalhousie University, Canada

Reviewed by:

Katsuhito Fujiu,

The University of Tokyo, Japan

Emiliano Medei,

Federal University of Rio de Janeiro,

Brazil

Justin Deniset

University of Calgary, Canada

*Correspondence:

Jianjun Tang

tom200210@csu.edu.cn

Shenghua Zhou

zhoushenghua@csu.edu.cn

tThese authors have contributed equally to this work

Specialty section:

This article was submitted to

Cardiac Electrophysiology,

a section of the journal

Frontiers in Physiology

Received: 25 June 2020

Accepted: 11 August 2020

Published: 23 September 2020

Citation:

Chen M, Li X, Wang S, Yu L,

Tang $J$ and Zhou S (2020) The Role

of Cardiac Macrophage

and Cytokines on Ventricular

Arrhythmias. Front. Physiol. 11:1113.

doi: 10.3389/fphys.2020.01113

\section{The Role of Cardiac Macrophage and Cytokines on Ventricular Arrhythmias}

\author{
Mingxian Chen ${ }^{1+}$, Xuping $\mathrm{Li}^{1+}$, Songyun Wang ${ }^{2}$, Lilei $\mathrm{Yu}^{2}$, Jianjun Tang ${ }^{1 *}$ and \\ Shenghua Zhou ${ }^{1 *}$ \\ ${ }^{1}$ The Second Xiangya Hospital, Central South University, Changsha, China, ${ }^{2}$ Department of Cardiology, Renmin Hospital
of Wuhan University, Wuhan, China
}

In the heart, cardiac macrophages have widespread biological functions, including roles in antigen presentation, phagocytosis, and immunoregulation, through the formation of diverse cytokines and growth factors; thus, these cells play an active role in tissue repair after heart injury. Recent clinical studies have indicated that macrophages or elevated inflammatory cytokines secreted by macrophages are closely related to ventricular arrhythmias (VAs). This review describes the role of macrophages and macrophage-secreted inflammatory cytokines in ventricular arrhythmogenesis.

Keywords: macrophage, cytokines, electrophysiology, ventricular arrhythmias, connexin43

\section{ORIGIN AND FUNCTIONS OF CARDIAC MACROPHAGES}

Tissue-resident macrophages have been observed in various organs, including the heart, brain, liver, and lung, and originate from the embryonic lineage, which is different from that of monocytic progenitors (Ginhoux et al., 2010; Davies et al., 2013; Moore et al., 2013). Cardiac macrophages maintain a homeostatic population through their self-proliferative properties and are independent of blood monocyte-derived macrophages. Recently, in steady-state conditions, two resident cardiac macrophage subsets, MHC-IIlowCCR2- and MHC-IIhighCCR2- cells, were identified by gene fate-mapping techniques. Under cardiac injury conditions, a third cardiac macrophage population, MHC-IIhighCCR2+ cells, was identified in the heart (Epelman et al., 2014; Honold and Nahrendorf, 2014). Cardiac CCR2- macrophages originate from the primitive yolk sac and are replenished through local proliferation, whereas CCR2+ macrophages originate from bone marrow-derived monocytes and repopulate through monocyte recruitment and proliferation. Resident CCR2- macrophages are involved in angiogenesis and cardiomyocyte proliferation. Bajpai et al. (2018) demonstrated that the human myocardium is populated by distinct subsets of CCR2 - macrophages, CCR2+ macrophages, and CCR2+ monocytes. Subsequently, Bajpai et al. (2019) showed that the depletion of resident cardiac CCR2- macrophages in a murine model of myocardial infarction increased the infarct area, reduced left ventricular (LV) systolic function, and aggravated LV remodeling. Interestingly, a recent study published in Cell demonstrated that resident macrophages in the steady-state heart facilitated electrical conduction, thus highlighting a novel concept regarding the potential role of cardiac macrophages in modulating cardiac electrical function (Hulsmans et al., 2017).

Under myocardial inflammatory conditions, monocyte-derived macrophages are recruited to the heart and characterized as MHC-IIhighCCR2+ cells. Mouse blood monocytes have been divided into Ly6C ${ }^{\text {hi }}$ and Ly6Clow (Robbins et al., 2012; Yap et al., 2019). Ly6C ${ }^{h i}$ monocytes induce excessive monocytosis, accumulate in the injured area, and differentiate into macrophages. Ly6 $\mathrm{C}^{\text {low }}$ monocytes, derived from pro-inflammatory Ly $6 \mathrm{C}^{\text {High }}$ cells, are less recruited than their 
Ly6C $\mathrm{C}^{\text {High }}$ counterparts following MI. Ly6C $\mathrm{C}^{\text {low }}$ monocytes are responsible for patrolling and tissue injury repair. However, human peripheral blood monocytes have 3 phenotypes: proinflammatory $\mathrm{CD} 14^{++} \mathrm{CD} 16^{-}$monocytes, anti-inflammatory $\mathrm{CD} 14^{+} \mathrm{CD} 16^{++}$monocytes with a function similar to Ly6 $\mathrm{C}^{\text {low }}$ monocytes, and proinflammatory CD $14{ }^{++} \mathrm{CD}_{16}{ }^{+}$monocytes secreting TNF- $\alpha$ (Heidt et al., 2014; van der Laan et al., 2014).

\section{MACROPHAGE ACTIVATION UNDER INFLAMMATORY CONDITIONS}

Macrophages are extremely heterogeneous and show adaptation of the phenotype and functions according to the surrounding microenvironment and aging (Gosselin et al., 2014; Pinto et al., 2014). Macrophage activation produces distinct functional phenotypes that are most commonly categorized as classically "inflammatory" macrophages and alternatively "anti-inflammatory" macrophages. This is a basic delineation of macrophage phenotypes but an overly simplistic view of macrophage behavior. Classically activated macrophages have proinflammatory properties, whereas alternatively activated macrophages are linked to cell proliferation and tissue repair (Nahrendorf et al., 2007; Biswas and Mantovani, 2010; Italiani and Boraschi, 2014). Classically activated macrophages secrete proinflammatory cytokines such as interferin-1 $\beta$ (IL-1 $\beta$ ), IL-6, IL-12, tumor necrosis factor- $\alpha$ (TNF- $\alpha$ ), and matrix metalloproteinase (MMP) and chemokines, which play a key role in host defense. Alternative activated macrophages produce cytokines, including transforming growth factor- $\beta$ (TGF- $\beta$ ), IL-10, enzyme arginase-1 in mice (ARG 1) and chemokines, which are involved in collagen formation and tissue repair.

After myocardial infarction (MI), macrophages are abundant in the infarcted area. Ly- $6 \mathrm{C}^{\text {High }}$ monocytes from the bone marrow and spleen are recruited to the infarcted zone and then differentiate into macrophages. The levels of this inflammatory subtype reach a peak approximately 3 days after injury (Heidt et al., 2014). Between days 5 and 7, macrophage populations are at their maximum within the infarct (Nian et al., 2004; Libby, 2013). Classically activated macrophages dominate the cell population of the infarcted zone. Classically activated macrophages produce proinflammatory cytokines, enhance the proinflammatory response and facilitate the breakdown of collagen. The reparative phase is characterized by phenotypic transition from inflammatory monocytes and macrophages (Ly-6C $\mathrm{C}^{\text {High }}$ monocytes and classically activated macrophages) to the anti-inflammatory subtypes (Ly-6C ${ }^{\text {low }}$ monocytes and alternatively activated macrophages) (Nahrendorf et al., 2007). The pool of cardiac macrophages is replenished as Ly-6C $\mathrm{C}^{\text {low }}$ monocytes are extensively recruited to the infarcted area. Ly-6C $\mathrm{C}^{\text {low }}$ monocytes mainly derive from pro-inflammatory Ly-6 $\mathrm{C}^{\text {High }}$ monocytes (Hilgendorf et al., 2014). After accumulation, Ly-6C ${ }^{\text {low }}$ monocytes are thereby differentiated into alternatively activated macrophages. Alternatively activated macrophages release IL-10, which inhibits the proinflammatory effects of classically activated macrophages, and TGF- $\beta$, which promotes tissue remodeling and angiogenesis (Lavine et al., 2014). Fei et al. (2019) demonstrated that activated macrophages directly connected to cardiomyocytes, thereby prolonging the action potential duration (APD), and ultimately led to APD heterogeneity and post-MI arrhythmias via gap junctions. This finding suggests that macrophages directly participate in ventricular arrhythmias after myocardial injury.

\section{MACROPHAGE-RELATED INFLAMMATION AND VENTRICULAR ARRHYTHMIAS}

Previously, several clinical studies provided evidence that increased inflammatory cytokines are closely associated with cardiac arrhythmias (Lewek et al., 2014). These results suggested that inflammation affects the initiation and progression of VAs. The proarrhythmic effects involve substrate-triggered cardiac electrical and structural remodeling. Inflammation contributes to the occurrence of ectopic-triggered activity and re-entry (Wakili et al., 2011; Dobrev et al., 2012; Aulin et al., 2015).

Electrophysiological changes (ion channel disturbance, early and late afterdepolarizations), as well as gap junction remodeling and enhanced myocardial fibrosis, are immunerelated mechanisms responsible for cardiac arrhythmias. Macrophage-dependent and macrophage-independent inflammation, including cytokine processes, serves as the basis of proinflammatory-induced VAs. Inflammation in the heart can also directly result in fluctuations in membrane potential. VAs can be triggered by early afterdepolarizations (EADs) and delayed afterdepolarizations (DADs). EAD results from the reduced function of potassium channels or the increased function of calcium or sodium channels. Abnormal intracellular $\mathrm{Ca}^{2+}$ handling, such as sarcoplasmic reticulum (SR) overload and uncontrolled $\mathrm{Ca}^{2+}$ leak, contributes to DAD (Kao et al., 2010; Chilukoti et al., 2013). Furthermore, gap junctions (GPs) are cell-to-cell pathways mediating electrical and chemical signal exchange between adjacent myocytes. GPs can transmit an orderly wave of electrical excitation. In the ventricles, gap junction remodeling, including connexin43 (Cx43) reduction, $\mathrm{Cx} 43$ dephosphorylation and $\mathrm{Cx} 43$ lateralization in pathological conditions, produces arrhythmia substrates (Duffy, 2012). The inflammatory response in the local heart area may play a crucial role in GP remodeling. Finally, excessive fibrosis or cardiac sarcoid infiltrated with abundant macrophages produces VAs not only by the mechanism of triggered substrate but also by re-entry (Okada et al., 2018). On the one hand, Haider et al. (2019) showed that cardiac macrophages can develop a fibroblast-like phenotype and directly contribute to the formation of fibrosis after myocardial infarction. On the other hand, macrophage-derived cytokines activate fibroblasts and produce cardiac fibrosis (Jung et al., 2017; Shimodaira et al., 2018; Abe et al., 2019). Cardiac fibrosis complicates electrical impulse propagation, slows conduction velocity, and forms unidirectional conduction blocks (Rohr et al., 1997; Rohr, 2012). Inflammation is also related to tissue repair after injury. 
Tissue repair is accompanied by parenchymal cell regeneration and finally fibrous tissue formation, namely, scar formation (Klein et al., 2000; Said et al., 2011; Gorenek et al., 2014; Vonderlin et al., 2019).

\section{MACROPHAGE-INDUCED CARDIAC SYMPATHETIC REMODELING AND VENTRICULAR ARRHYTHMIAS}

Sympathetic overactivity and structural remodeling play a critical role in ventricular arrhythmogenesis (Podrid et al., 1990; Shen and Zipes, 2014; Kalla et al., 2016). In an experimental study, sympathetic nerve stimulation caused a change in ventricular electrophysiology, reduced the ventricular fibrillation threshold and triggered Vas (Mantravadi et al., 2007; Ng et al., 2009). Although it is difficult to induce VAs in mice, norepinephrine (NE) injected into the epicardial tissue of guinea pigs elicits triggered automaticity, and computational modeling identified a $\mathrm{Ca}^{2+}$ overload mechanism in cardiac ventricular electrophysiology. This finding supports the hypothesis that heterogeneity or gradients of sympathetic activation are proarrhythmic (Vaseghi et al., 2012). A growing body of work has demonstrated that macrophages can contribute to sympathetic hyperactivity. Levick et al. (2010) showed that substance $\mathrm{P}$ released by sympathetic afferent fibers could bind to the neurokinin-1 receptor of macrophages to induce the production of macrophage-derived angiotensin II. Angiotensin II could further stimulate the terminus of sympathetic efferent fibers and then increase the production of norepinephrine. Thus, macrophages play a critical role in mediating VAs relevant to sympathetic activity by enhancing the production of angiotensin II (Levick et al., 2010). Furthermore, peripheral proinflammatory factors produced by macrophages, such as IL-1 $\beta$, IL-6, and TNF- $\alpha$, can transmit signals to the brain by the circulation or through afferent fibers, which in turn activate the sympathetic nervous system (SNS).

A proarrhythmic substrate is usually formed by regional myocardial remodeling, as well as heterogeneity of sympathetic innervation (Barber et al., 1983; Tomaselli and Zipes, 2004). The heterogeneity of sympathetic innervation is called nerve sprouting (Cao et al., 2000; Chen et al., 2001). Emerging data have indicated that cardiac sympathetic sprouting and cardiac electrical remodeling are involved in the post-MI remodeling process (Zipes and Rubart, 2006; Wang et al., 2012). Sympathetic nerve sprouting may produce electrical and structural remodeling following AMI, resulting in electrophysiological instabilities and finally inducing VAs. In fact, studies have shown that the inhibition of sympathetic nerve sprouting induced by MI can exert antiarrhythmogenic effects (Wernli et al., 2009; Yang et al., 2016; Yin et al., 2016; Hu et al., 2019). Sympathetic nerve remodeling is a complex pathophysiological process, and sympathetic nerve sprouting is closely associated with the inflammatory reaction and is primarily present at the infarct border zone, where abundant macrophages and macrophagederived cytokines are observed. Macrophages could promote sympathetic hyperinnervation via the regulation of nerve growth factor (NGF) expression (Yin et al., 2016). Atorvastatin could effectively improve cardiac sympathetic nerve remodeling by modulating macrophage polarization (Yang et al., 2016). It was recently demonstrated that inhibiting miR-155 can downregulate NGF expression by decreasing M1 macrophage polarization, subsequently impairing sympathetic nerve remodeling and VAs induced by acute myocardial infarction (Wernli et al., 2009; Hu et al., 2019).

Sympathetic nervous system can affect macrophages at both the systemic and regional levels (Chen et al., 2016). SNS fibers innervate the primary and second lymphoid organs (bone marrow, thymus, spleen, and lymph nodes) and are capable of modulating immune functions. The SNS contributes to the differentiation, maturation, recruitment, and regulation of macrophages. Moreover, the SNS directly innervates the target lesion, plays a proinflammatory role and promotes M1 polarization. Norepinephrine released from sympathetic nerve endings could bind to $\alpha$ - or $\beta$-adrenergic receptors expressed on immune cells ( $\mathrm{T}$ cells, B cells, natural killer cells, and macrophages). This response gives rise to a cascade of events, including the production of proinflammatory cytokines and recruitment of leukocytes. Therefore, these changes might form a vicious circle between SNS activity and M1 polarization, both contributing to ventricular arrhythmogenesis.

\section{CYTOKINES SECRETED BY MACROPHAGE AND VENTRICULAR ARRHYTHMIAS}

Macrophages secrete multiple cytokines. Proinflammatory factors produced by macrophages, such as IL- $1 \beta$, IL- 6 , TNF- $\alpha$, and matrix metalloproteins (MMPs), can regulate cardiac SNS activity, form a proarrhythmic substrate and directly affect myocardial electrophysiology (Hirayama et al., 2017). VAs might be induced by cytokines derived from macrophages in both acute and chronic diseases. It was shown that cytokines derived from macrophages following acute MI could target cardiac myocytes and induce electrophysiological remodeling, including a reduction in repolarizing $\mathrm{K}^{+}$currents, $\mathrm{Cx} 43$ expression and intracellular $\mathrm{Ca}^{2+}$ mishandling (Pinto and Boyden, 1999; Francis Stuart et al., 2016). These changes could provide the trigger and substrate for ventricular arrhythmias (De Jesus et al., 2017). In the case of chronic inflammation, a growing body of clinical evidence has demonstrated that the serum concentration of macrophage-derived cytokines was significantly higher in post-MI patients with ventricular arrhythmias than in post-MI patients without ventricular arrhythmias (Streitner et al., 2007, 2009; Francis Stuart et al., 2016). Furthermore, Monnerat et al. (2016) experimentally demonstrated that IL- $\beta$ derived from cardiac macrophages could trigger ventricular arrhythmias in mice. Even in the absence of cardiac injury, systemic inflammation was found to be related to an increased risk for ventricular arrhythmias. Extracardiac injury was also shown to enhance macrophage-related inflammation in the heart. VAs are also one of the most critical complications after renal or brain injury. Renal ischemia reperfusion increased 
susceptibility to ventricular arrhythmias depending on activation of NLRP3-CASP1-IL-1 $\beta$; accordingly, this effect was inhibited by macrophage depletion (Alarcon et al., 2019). Rheumatoid arthritis is a chronic inflammatory disease, and increased serum concentrations of macrophage-derived cytokines were detected. Increased susceptibility to VAs was also found in patients with rheumatoid arthritis (Lazzerini et al., 2017a). The role of individual cytokines on VAs is listed below in detail (Figure 1).

\section{IL-1 $\beta$ and Ventricular Arrhythmias}

IL-1, as an activating factor of endothelial cells, could regulate and initiate inflammatory responses. The IL-1 family is a group of 11 cytokines. IL- $1 \alpha$ and IL- $1 \beta$ are the most studied members because of their early discovery and significant proinflammatory effects. IL-1 $\beta$ is synthesized as a precursor protein after stimulation by activated monocyte-macrophages (Guillén et al., 1995; Saxena et al., 2013).

IL- $1 \beta$ is a crucial regulator in the inflammatory response after $\mathrm{MI}$ and is involved in the modulation of immune cell recruitment, cytokine production, and extracellular matrix turnover. Clinical studies have revealed that the levels of IL-1 $\beta$ in both tissue and plasma are significantly increased in patients with VAs. These results suggest that in addition to the above biological functions, IL-1 $\beta$ might be an important mediator of ion channel remodeling, thereby producing Vas (Fernández-Sada et al., 2017; Abbate et al., 2020).

Emerging evidence has demonstrated that IL-1 $\beta$ can directly affect the electrical properties of cardiomyocytes (CMs). IL$1 \beta$ resulted in changes in $\mathrm{Ca}^{2+}$ handling (Alarcon et al., 2019). Li and Rozanski (1993) found an increase in $I_{C a L}$ in guinea pig myocytes, inducing the prolongation of the action potential duration (APD) and the effective refractory period (ERP). Moreover, Liu et al. observed that IL-1 $\beta$ decreased the responsiveness of $\mathrm{I}_{\mathrm{CaL}}$ to $\beta$-adrenergic stimulation (Liu et al., 1999). IL-1 $\beta$, synergistic with TNF- $\alpha$ application, also affected $\mathrm{SR} \mathrm{Ca}^{2+}$ release and reuptake in rat ventricular myocytes, which contributed to the depressed $\mathrm{Ca}^{2+}$ transient and contractility (Duncan et al., 2010). Spontaneous SR Ca ${ }^{2+}$ release may increase the susceptibility to arrhythmias, leading to cell depolarization. Recently, Monnerat et al. (2016) showed that IL-1 $\beta$ produced by macrophages derived from the hearts of individuals with diabetes mellitus could directly target cardiomyocytes to induce VAs. IL-1 $\beta$ then induces a decrease in the Ito current and an increase in $\mathrm{Ca}^{2+}$ sparks, resulting in increased electrical vulnerability to arrhythmias. In a mouse model, De Jesus et al. (2017) found that IL-1 $\beta$ inhibition improved conduction velocity, reduced APD dispersion, improved intracellular $\mathrm{Ca}^{2+}$ handling, decreased the transmembrane potential and the magnitude of the $\mathrm{Ca}^{2+}$ alternans, and thus reduced spontaneous and inducible Vas (Su et al., 2018).

IL-1 $\beta$ contributes to electrical function not only through direct effects but also indirect effects on VAs. Wang et al. confirmed that IL-1 $\beta$ injection into the left stellate ganglion (LSG) could increase sympathetic activity and the occurrence of VA. IL-1 $\beta$ injection induced cardiac electrical remodeling, and this response was attenuated by IL-1Ra preinjection (Wang et al., 2017). Cardiac fibrotic substrate and $\mathrm{Cx} 43$ remodeling are important mediators responsible for the heterogeneity in ventricular conduction for reentry. Considerable experimental data have shown that excess fibrosis promotes ectopic triggers in the hearts of aged rats and rabbits (Bapat et al., 2012; Wang et al., 2017). This kind of proarrhythmic substrate produced early afterdepolarizations, triggered activity and reduced conduction velocity (Bapat et al., 2012). Previous study has shown that IL- $1 \beta$ plays an important role in the formation of fibrotic substrates and is implicated in Cx43 remodeling, induced cell-cell uncoupling, lateralization, and degradation (Baum et al., 2012).

Thus, macrophage-derived IL-1 $\beta$ during myocardial healing could induce deleterious electrophysiological consequences.

\section{TNF- $\alpha$ and Ventricular Arrhythmias}

TNF- $\alpha$ is one of the most important inflammatory factors and is mainly secreted by activated macrophages. Myocardial TNF$\alpha$ expression was significantly upregulated post AMI (Feldman et al., 2000). TNF- $\alpha$ has widespread biological effects on cell proliferation, differentiation, apoptosis and inflammatory reactions (Libby et al., 2002; MacEwan, 2002). Clinical evidence confirmed that the elevation of plasma TNF- $\alpha$ in patients with AMI was closely related to the occurrence of Vas (Halawa et al., 1999; Eskandarian et al., 2013). Experimental studies also showed that transgenic animals with TNF- $\alpha$ overexpression are prone to severe Vas (London et al., 2003; Chen et al., 2010).

TNF- $\alpha$ may alter the electric activity of cardiac myocytes by different mechanisms and finally induce Vas (Petkova-Kirova et al., 2006). TNF- $\alpha$ modulates cardiac $K^{+}$channels. TNF- $\alpha$ can induce a significant reduction in Ito density, modify Ito inactivation, and downregulate $K_{v 4.2}$ protein expression. TNF$\alpha$ could inhibit the cardiac delayed rectifier $\mathrm{K}$ current via the protein kinase A (PKA) pathway (Hatada et al., 2006). Furthermore, TNF- $\alpha$ appears to have a significant impact on cellular $\mathrm{Ca}^{2+}$ release and uptake. This molecule disrupted cellular $\mathrm{Ca}^{2+}$ cycling, which increased the probability of proarrhythmic spontaneous $\mathrm{Ca}^{2+}$ release from the SR, which may contribute to the increased incidence of arrhythmia in sepsis in isolated rat ventricular myocytes (Duncan et al., 2010). It was found that the regulation of $\mathrm{Ca}^{2+}$ inflow of cardiac myocytes was achieved by the phospholipase A2/arachidonic acid (PLA2/AA) pathway (Amadou et al., 2002).

Slowed myocardial conduction velocity (CV) increases the risk of re-entrant excitation, predisposing patients to cardiac arrhythmia. CV is determined by the ion channel and cellular interconnections. George et al. (2017) demonstrated that TNF $\alpha$ could reduce $\mathrm{CV}$ by altering electrical coupling between myocytes in guinea pig hearts. The effects of TNF- $\alpha$ on gap junction coupling have been extensively studied. TNF- $\alpha$ alters Cx43 expression, reduces $\mathrm{Cx} 43$ phosphorylation, and alters $\mathrm{Cx} 43$ redistribution, which is important in modulating $\mathrm{Cx} 43$ channel conductance (Fernandez-Cobo et al., 1999; Sawaya et al., 2007; George et al., 2017).

\section{IL-6 and Ventricular Arrhythmias}

IL-6 is also involved in multiple biological effects, including cardiomyocyte response to injury (Yang et al., 2004; 


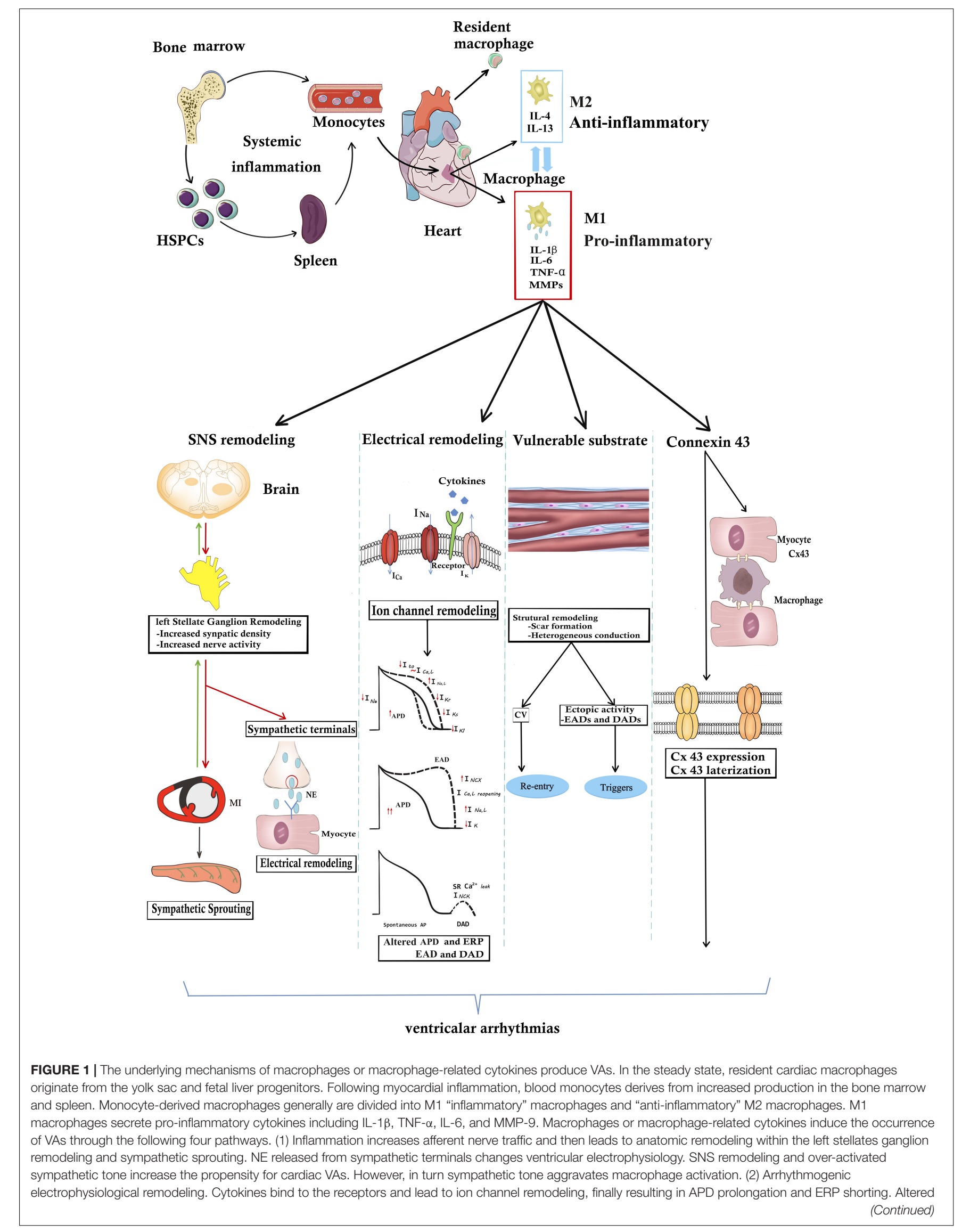




\section{FIGURE 1 | Continued}

ionic currents contribute to EAD and DAD increasing the arrhythmogenic substrate. (3) Macrophage-related cytokines induce the scar formation and CV heterogeneity, resulting in re-entry. Structural remodeling also induces EDA and DAD, producing arrhythmogenic triggers. (4) Inflammation directly changes the Cx43 structural and functional remodeling. However, whether inflammation promotes Cx43 remodeling between myocytes and macrophages still remain unknown. It needs further study. APD, action potential; Cx43, connexin43; CV, conduction velocity; DAD, delayed afterdepolarization; EDA, early afterdepolarization; IL-1 $\beta$, HSPCs, hematopoiesis precursor cells; interleukin 1 $\beta$; IL-6, interleukin 6; MMP-9, matrix metalloproteinases 9; NE, norepinephrine; TNF- $\alpha$, tumor necrosis factor- $\alpha$.

Alí et al., 2018). Serum levels of IL-6 and its mRNA and protein expression in cardiac tissues are significantly increased in patients with cardiac diseases, including heart failure, myocarditis, septic cardiomyopathy, myocardial infarction, and cardiac myxoma (Ikeda et al., 1992). IL-6 plays a critical role in the pathophysiology of these cardiac disorders. A study demonstrated that elevated serum IL-6 levels were associated with an increase in susceptibility to spontaneous ventricular tachyarrhythmia in patients with coronary artery disease (Streitner et al., 2007). Recently, accumulating data obtained from patients with myocarditis/endocarditis and systemic autoimmune diseases (Ukena et al., 2011), particularly rheumatoid arthritis (Lazzerini et al., 2015a) and other connective tissue diseases, demonstrated that circulating IL-6 levels are elevated in these patients. Increased IL-6 levels are correlated with vulnerability to QT interval prolongation, which contributes prominently to arrhythmic events and torsade de pointes (TdP) (Adlan et al., 2015; Lazzerini et al., 2015b; Lazzerini et al., 2017b). A study also indicated that IL-6 possessed a potential direct electrophysiological effect on ion channels that can alter the APD and QTc interval (Aromolaran et al., 2018).

Emerging experimental evidence showed that IL-6 could regulate the electrophysiological properties of cardiomyocytes. Previous data suggest that IL-6 may play a critical role in contributing to the modulation of $I_{C a, L}$ and $I_{K}$ currents, and both factors are active contributors to cardiac instabilities. Hagiwara et al. (2007) found that IL-6 could regulate $\mathrm{I}_{\mathrm{CaL}}$ and density. After acute (30 $\mathrm{min}$ ) exposure to IL-6 and soluble IL-6 receptor (IL-6R), the $\mathrm{I}_{C a, L}$ density in mouse ventricular myocytes was significantly increased, which was strongly associated with LQTS. IL-6 was also shown to cause QT prolongation by suppressing $I_{K r}$. Ademuyiwa et al. demonstrated that IL-6 alone or in combination with soluble IL-6R could inhibit the $I_{K r}$ peak and result in the prolongation of APD via Janus kinase (JAK) pathway activation, forming the basis for the observed clinical QT interval prolongation (Aromolaran et al., 2018). In vitro studies have shown that IL-6 suppresses peak cytosolic intracellular $\mathrm{Ca}^{2+}$ and cell contraction of cardiomyocytes within minutes due to activation of $\mathrm{Ca}^{2+}$-dependent nitric oxide synthetase (Kinugawa et al., 1994; Yu et al., 2003; Monnerat et al., 2016).

\section{MMP-9 and Ventricular Arrhythmias}

In hearts, myocardial injury was shown to activate macrophages to increase MMP-9 secretion. Clinical data have shown that serum MMP-9 levels are significantly elevated in patients with cardiac dysfunction (Li et al., 1998; Thomas et al., 1998; Sivakumar et al., 2008) and are closely associated with increased VAs and sudden cardiac death (Flevari et al., 2012; Hästbacka et al., 2012; Turkdogan et al., 2017), while the downregulation of MMP-9 by gene modification or pharmacological inhibition significantly reduced the incidence of VAs in a mouse model (Weng et al., 2016). The above evidence indicates that MMP-9 plays a critical role in the pathophysiology of VAs.

Experimental data demonstrated that MMP-9 was mainly involved in the regulation of cardiomyocyte electrophysiological properties via the formation of cardiac fibrosis, gap junction remodeling and calcium homeostasis (Weng et al., 2016). Cardiac fibrosis, $\mathrm{Cx} 43$ reduction and lateralization are prerequisites of ventricular conduction heterogeneity for re-entry. MMP-9 is a key regulator of the reparative phases of post-MI healing and a necessary modulator for proper scar formation. MMP9 could enhance myocardial remodeling, result in excessive extracellular matrix degradation, increase myocardial fibrosis, and thus contribute to re-entry and eventually lead to VAs. MMP-9 might also degrade $\mathrm{Cx} 43$, which is required for proper cell-cell electrical coupling (Fontes et al., 2012; Nguyen et al., 2014). Mukherjee et al. (2010) demonstrated that MMP-9 activity corresponded to increased Cx43 lateralization and reduced conduction velocity. Excessive MMP-9 could disrupt normal cell-cell electrical communication post-MI. A recent study showed that MMP-9 could increase $\mathrm{Ca}^{2+}$ leakage from the SR, which could depolarize cardiomyocytes and trigger fatal arrhythmia. MMP-9 also decreases CD36 and increases PKA activity. Activated PKA subsequently triggers ryanodine receptor 2 (RyR2) phosphorylation, leading to a higher probability of RyR pore opening, followed by increased calcium leakage. An increase in calcium sparks activates an arrhythmogenic depolarizing inward $\mathrm{Na}^{+} / \mathrm{Ca}^{2+}$ exchange current, which causes delayed afterdepolarizations and triggers VAs and sudden cardiac death (Marx et al., 2000; Dobrev and Wehrens, 2014; Wehrens et al., 2004; DeLeon-Pennell et al., 2016).

\section{THE CONNEXINS BETWEEN MACROPHAGES AND CARDIOMYOCYTES ON VENTRICULAR ARRHYTHMIAS}

In addition to antigen presentation, phagocytosis and immunomodulation, cardiac macrophages have been proven to directly induce cardiac electrophysiological changes. This finding substantially changed our previous knowledge of macrophage function. Previous studies have shown that the transmembrane potential is $-26 \mathrm{mV}$ for mouse macrophages and $-18 \mathrm{mV}$ for guinea pig macrophages (Dos Reis et al., 1979). Macrophages present several different types of $\mathrm{K}^{+}$ and $\mathrm{Cl}^{-}$channels in the membrane. Membrane potential 
and ionic conductance regulate many cell functions, including transmembrane signaling, phagocytosis, secretion, and motility (Udagawa, 2003; Pinto, 2017). However, previous data did not show that macrophages could directly modulate cardiac electrophysiology.

A recent study reported by Hulsmans et al. (2017) demonstrated that cardiac resident macrophages are integral for normal heart rhythm. These researchers found that cardiac macrophages are highly abundant at the atrioventricular node (AVN) in mice and humans. They found that macrophages connect to cardiomyocytes at the AVN via $\mathrm{Cx} 43$, forming punctate junctions between macrophages and cardiomyocytes. Cx43-mediated macrophage-CM coupling served as electrical coupling of the two cell types. In an in vitro study, cocultures of macrophages and mouse neonatal AVN CMs also established $\mathrm{Cx} 43$ coupling between the two cell types. The researchers further showed that depolarization of macrophages improved AVN conductance. However, depletion of macrophages resulted in AVN block, resulting in arrhythmia.

In the injured heart, the number of cardiac macrophages is significantly increased compared with that in the uninjured heart. Considering that resident macrophages might participate in cardiac electrophysiology through $\mathrm{Cx} 43$ between macrophages and myocytes, it would be interesting to investigate whether monocyte-derived macrophages recruited to the myocardium under inflammatory conditions also connect to myocytes by Cx43, affecting cardiomyocyte electrophysiology and resulting in VAs. Fei et al. (2019) found that proinflammatory macrophages formed gap junctions with cardiomyocytes and accumulated in MI border zones 3 days post-MI. These researchers further demonstrated that non-inflammatory macrophages connected with myocardiocytes could shorten $\mathrm{APD}_{90}$, yet proinflammatory macrophages could prolong $\mathrm{APD}_{90}$ in an in vitro coculture experiment. This finding indicates that the Cx43 connection between macrophages and cardiomyocytes leads to APD heterogeneity and post-MI arrhythmias. Therefore, targeting macrophage-CM coupling could be a potential useful target when treating inflammation-associated conduction abnormalities.

\section{REFERENCES}

Abbate, A., Toldo, S., Marchetti, C., Kron, J., Van Tassell, B. W., and Dinarello, C. A. (2020). Interleukin-1 and the inflammasome as therapeutic targets in cardiovascular disease. Circ. Res. 126, 1260-1280. doi: 10.1161/circresaha.120. 315937

Abe, H., Takeda, N., Isagawa, T., Semba, H., Nishimura, S., Morioka, M. S., et al. (2019). Macrophage hypoxia signaling regulates cardiac fibrosis via Oncostatin M. Nat. Commun. 10:2824.

Adlan, A. M., Panoulas, V. F., Smith, J. P., Fisher, J. P., and Kitas, G. D. (2015). Association between corrected QT interval and inflammatory cytokines in rheumatoid arthritis. J. Rheumatol. 42, 421-428. doi: 10.3899/jrheum.140861

Alarcon, M. M. L., Trentin-Sonoda, M., Panico, K., Schleier, Y., Duque, T., MorenoLoaiza, O., et al. (2019). Cardiac arrhythmias after renal I/R depend on IL-1 $\beta$. J. Mol. Cell Cardiol. 131, 101-111. doi: 10.1016/j.yjmcc.2019.04.025

Alí, A., Boutjdir, M., and Aromolaran, A. S. (2018). Cardiolipotoxicity, inflammation, and arrhythmias: role for Interleukin-6 molecular mechanisms. Front. Physiol. 9:1866. doi: 10.3389/fphys.2018.01866

\section{FUTURE THERAPEUTIC DIRECTION AND CONCLUSION}

Macrophages appear to play direct and indirect roles in the occurrence of VAs. Activation of cardiac macrophages induces VAs through sympathetic nerve sprouting, proinflammatory cytokine production, and the direct influence of cardiac electrophysiology. Previously, inflammation has always been treated as an epiphenomenon and not suitable as a target for intervention. However, based on experimental and clinical evidence, anti-inflammatory therapy targeting the inflammatory cytokines contributed to a benefit from cardiovascular diseases (Ridker et al., 2017; Wang et al., 2017). Canakinumab Antiinflammatory Thrombosis Outcomes Study (CANTOS) has been provided the evidence that IL- $1 \beta$ treated as a target and reduced major cardiovascular events. It opens a landscape of cardiovascular diseases therapy (Ridker et al., 2017). Therefore, immunotherapy for therapeutic interventions, targeting these functional elements of macrophages and cytokines blockade, are likely to be a promising new research avenue and might be valuable for the purpose of developing new therapeutics to reduce VAs.

\section{AUTHOR CONTRIBUTIONS}

MC, XL, and SW participated in the study design and drafted the manuscript. SZ and MC responsible for writing the manuscript. LY, JT, and SZ participated in the overall editing and approval of the manuscript. All authors contributed to the article and approved the submitted version.

\section{FUNDING}

The authors would like to thank LY, Minjia Xiao, and Zhuo Wang for modification. Financial support was obtained from the National Natural Science Foundation of China Nos. 81270257 and 81800302 and Provincial Natural Science Foundation of Hunan No. 2019JJ50871.

Amadou, A., Nawrocki, A., Best-Belpomme, M., Pavoine, C., and Pecker, F. (2002). Arachidonic acid mediates dual effect of TNF-alpha on $\mathrm{Ca} 2+$ transients and contraction of adult rat cardiomyocytes. Am. J. Physiol. Cell Physiol. 282, C1339-C1347.

Aromolaran, A. S., Srivastava, U., Alí, A., Chahine, M., Lazaro, D., El-Sherif, N., et al. (2018). Interleukin-6 inhibition of hERG underlies risk for acquired long QT in cardiac and systemic inflammation. PLoS One 13:e0208321. doi: 10.1371/ journal.pone.0208321

Aulin, J., Siegbahn, A., Hijazi, Z., Ezekowitz, M. D., Andersson, U., Connolly, S. J., et al. (2015). Interleukin-6 and C-reactive protein and risk for death and cardiovascular events in patients with atrial fibrillation. Am. Heart J. 170, 1151-1160. doi: 10.1016/j.ahj.2015.09.018

Bajpai, G., Bredemeyer, A., Li, W., Zaitsev, K., Koenig, A. L., Lokshina, I., et al. (2019). Tissue resident CCR2- and CCR2 + cardiac macrophages differentially orchestrate monocyte recruitment and fate specification following myocardial injury. Circ. Res. 124, 263-278. doi: 10.1161/circresaha.118.314028

Bajpai, G., Schneider, C., Wong, N., Bredemeyer, A., Hulsmans, M., Nahrendorf, M., et al. (2018). The human heart contains distinct macrophage subsets with 
divergent origins and functions. Nat. Med. 8, 1234-1245. doi: 10.1038/s41591018-0059-x

Bapat, A., Nguyen, T. P., Lee, J. H., Sovari, A. A., Fishbein, M. C., Weiss, J. N., et al. (2012). Enhanced sensitivity of aged fibrotic hearts to angiotensin II- and hypokalemia-induced early afterdepolarization-mediated ventricular arrhythmias. Am. J. Physiol. Heart Circ. Physiol. 302, H2331-H2340.

Barber, M. J., Mueller, T. M., Henry, D. P., Felten, S. Y., and Zipes, D. P. (1983). Transmural myocardial infarction in the dog produces sympathectomy in noninfarcted myocardium. Circulation 67, 787-796. doi: 10.1161/01.cir.67. 4.787

Baum, J. R., Dolmatova, E., Tan, A., and Duffy, H. S. (2012). Omega 3 fatty acid inhibition of inflammatory cytokine-mediated Connexin 43 regulation in the heart. Front. Physiol. 3:272. doi: 10.3389/fphys.2012.00272

Biswas, S. K., and Mantovani, A. (2010). Macrophage plasticity and interaction with lymphocyte subsets: cancer as aparadigm. Nat. Immunol. 11, 889-896. doi: 10.1038/ni.1937

Cao, J. M., Chen, L. S., KenKnight, B. H., Ohara, T., Lee, M. H., Tsai, J., et al. (2000). Nerve sprouting and sudden cardiac death. Circ. Res. 86, 816-821.

Chen, M. X., Liu, Q. M., and Zhou, S. H. (2016). The networks between the sympathetic nervous system and immune system in atherosclerosis. J. Am. Coll. Cardiol. 68, 431-432.

Chen, P. S., Chen, L. S., Cao, J. M., Sharifi, B., Karagueuzian, H. S., and Fishbein, M. C. (2001). Sympathetic nerve sprouting, electrical remodeling and the mechanisms of sudden cardiac death. Cardiovasc. Res. 50, 409-416. doi: 10. 1016/s0008-6363(00)00308-4

Chen, Y., Chen, Z. J., Liao, Y. H., Cao, Z., Xia, J. D., Yang, H., et al. (2010). Effect of tumor necrosis factor- $\alpha$ on ventricular arrhythmias in rats with acute myocardial infarction in vivo. World J. Emerg. Med. 1, 53-58.

Chilukoti, R. K., Mostertz, J., Bukowska, A., Aderkast, C., Felix, S. B., Busch, M., et al. (2013). Effects of irbesartan on gene expression revealed by transcriptome analysis of left Epelman atrial tissue in a porcine model of acute rapid pacing in vivo. Int. J. Cardiol. 168, 2100-2108. doi: 10.1016/j.ijcard.2013.01.007

Davies, L. C., Jenkins, J. S., Allen, J. E., and Taylor, P. R. (2013). Tissue-resident macrophages. Nat. Immunol. 14, 986-995.

De Jesus, N. M., Wang, L., Lai, J., Rigor, R. R., Francis Stuart, S. D., Bers, D. M., et al. (2017). Antiarrhythmic effects of interleukin 1 inhibition after myocardial infarction. Heart Rhythm 14, 727-736. doi: 10.1016/j.hrthm.2017.01.027

DeLeon-Pennell, K. Y., Tian, Y., Zhang, B., Cates, C. A., Iyer, R. P., Cannon, P., et al. (2016). CD36 is a matrix metalloproteinase-9 substrate that stimulates neutrophil apoptosis and removal during cardiac remodeling. Circ. Cardiovasc. Genet. 9, 14-25. doi: 10.1161/circgenetics.115.001249

Dobrev, D., Carlsson, L., and Nattel, S. (2012). Novel molecular targets for atrial fibrillation therapy. Nat. Rev. Drug Discov. 11, 275-291. doi: 10.1038/nrd3682

Dobrev, D., and Wehrens, X. H. (2014). Role of RyR2 phosphorylation in heart failure and arrhythmias: controversies around ryanodine receptor phosphorylation in cardiac disease. Circ. Res. 114, 1311-1319. doi: 10.1161/ circresaha.114.300568

Dos Reis, G. A., Persechini, P. M., Ribeiro, J. M., and Oliveira-Castro, G. M. (1979). Electrophysiology of phagocytic membranes. II. Membrane potential and induction of slow hyperpolarizations in activated macrophages. Biochim. Biophys. Acta 552, 331-340. doi: 10.1016/0005-2736(79)90287-6

Duffy, H. S. (2012). The molecular mechanisms of gap junction remodeling. Heart Rhythm 9, 1331-1334. doi: 10.1016/j.hrthm.2011.11.048

Duncan, D. J., Yang, Z., Hopkins, P. M., Steele, D. S., and Harrison, S. M. (2010). TNF-alpha and IL-1beta increase Ca2+ leak from the sarcoplasmic reticulum and susceptibility to arrhythmia in rat ventricular myocytes. Cell Calc. 47, 378-386. doi: 10.1016/j.ceca.2010.02.002

Epelman, S., Lavine, K. J., Beaudin, A. E., Sojka, D. K., Carrero, J. A., Calderon, B., et al. (2014). Embryonic and adult-derived resident cardiac macrophages are maintained through distinct mechanisms at steady state and during inflammation. Immunity 40, 91-104. doi: 10.1016/j.immuni.2013.11.019

Eskandarian, R., Ghorbani, R., and Asgary, Z. (2013). Relationship between leucocytosis and left ventricular ejection fraction in patients with acute myocardial infarction. Singapore Med. J. 54, 40-43. doi: 10.11622/smedj. 2013010

Fei, Y. D., Wang, Q., Hou, J. W., Li, W., Cai, X. X., Yang, Y. L., et al. (2019). Macrophages facilitate post myocardial infarction arrhythmias: roles of gap junction and KCa3.1. Theranostics 9, 6396-6411. doi: 10.7150/thno.34801
Feldman, A. M., Combes, A., Wagner, D., Kadakomi, T., Kubota, T., Li, Y. Y., et al. (2000). The role of tumor necrosis factor in the pathophysiology of heart failure. J. Am. Coll. Cardiol. 35, 537-544.

Fernandez-Cobo, M., Gingalewski, C., Drujan, D., and De Maio, A. (1999). Downregulation of connexin 43 gene expression in rat heart during inflammation. The role of tumour necrosis factor. Cytokine 11, 216-224. doi: 10.1006/cyto.1998.0422

Fernández-Sada, E., Torres-Quintanilla, A., Silva-Platas, C., García, N., Willis, B. C., Rodríguez-Rodríguez, C., et al. (2017). Proinflammatory cytokines are soluble mediators linked with ventricular arrhythmias and contractile dysfunction in a rat model of metabolic syndrome. Oxid. Med. Cell. Longev. 2017:7682569.

Flevari, P., Theodorakis, G., Leftheriotis, D., Kroupis, C., Kolokathis, F., Dima, K., et al. (2012). Serum markers ofderanged myocardial collagen turnover: their relation to malignant ventricular arrhythmias in cardioverter-defibrillator recipients with heart failure. Am. Heart J. 164, 530-537. doi: 10.1016/j.ahj.2012. 07.006

Fontes, M. S., van Veen, T. A., de Bakker, J. M., and van Rijen, H. V. (2012). Functional consequences of abnormal $\mathrm{Cx} 43$ expression in the heart. Biochim. Biophys. Acta 1818, 2020-2029. doi: 10.1016/j.bbamem.2011.07.039

Francis Stuart, S. D., De Jesus, N. M., Lindsey, M. L., and Ripplinger, C. M. (2016). The crossroads of inflammation, fibrosis, and arrhythmia following myocardial infarction. J. Mol. Cell Cardiol. 91, 114-122. doi: 10.1016/j.yjmcc.2015. 12.024

George, S. A., Calhoun, P. J., Gourdie, R. G., Smyth, J. W., and Poelzing, S. (2017). TNF $\alpha$ modulates cardiac conduction by altering electrical coupling between myocytes. Front. Physiol. 8:334. doi: 10.3389/fphys.2017.00334

Ginhoux, F., Greter, M., Leboeuf, M., Nandi, S., See, P., Gokhan, S., et al. (2010). Fate mapping analysis reveals that adult microglia derive from primitive macrophages. Science 330, 841-845. doi: 10.1126/science.1194637

Gorenek, B., Blomström Lundqvist, C., Brugada Terradellas, J., Camm, A. J., Hindricks, G., Huber, K., et al. (2014). Cardiac arrhythmias in acute coronary syndromes: position paper from the joint EHRA, ACCA, and EAPCI task force. Europace 16, 1655-1673. doi: 10.1093/europace/euu208

Gosselin, D., Link, V. M., Romanoski, C. E., Fonseca, G. J., Eichenfield, D. Z., Spann, N. J., et al. (2014). Environment drives selection and function of enhancers controlling tissue-specific macrophage identities. Cell 159, 13271340. doi: 10.1016/j.cell.2014.11.023

Guillén, I., Blanes, M., Gómez-Lechón, M. J., and Castell, J. V. (1995). Cytokine signaling during myocardial infarction: sequential appearance of IL-1 beta and IL-6. Am. J. Physiol. 269, R229-R235.

Hagiwara, Y., Miyoshi, S., Fukuda, K., Nishiyama, N., Ikegami, Y., Tanimoto, K., et al. (2007). SHP2-mediated signaling cascade through gp130 is essential for LIF-dependent I CaL, [Ca2+]i transient, and APD increase in cardiomyocytes. J. Mol. Cell Cardiol. 43, 710-716. doi: 10.1016/j.yjmcc.2007.09.004

Haider, N., Boscá, L., Zandbergen, H. R., Kovacic, J. C., Narula, N., GonzálezRamos, S., et al. (2019). Transition of macrophages to fibroblast-like cells in healing myocardial infarction. J. Am. Coll. Cardiol. 74, 3112-3120.

Halawa, B., Salomon, P., Jołda-Mydłowska, B., and Zyśko, D. (1999). Levels of tumor necrosis factor (TNF-alpha) and interleukin 6 (IL-6) in serum of patients with acute myocardial infarction. Polskie Archiwum Medycyny Wewnetrznej 101, 197-203.

Hästbacka, J., Tiainen, M., Hynninen, M., Kolho, E., Tervahartiala, T., Sorsa, T., et al. (2012). Serum matrix metalloproteinases in patients resuscitated from cardiac arrest. The association with therapeutic hypothermia. Resuscitation 83 , 197-201. doi: 10.1016/j.resuscitation.2011.07.036

Hatada, K., Washizuka, T., Horie, M., Watanabe, H., Yamashita, F., Chinushi, M., et al. (2006). Tumor necrosis factor-alpha inhibits the cardiac delayed rectifier $\mathrm{K}$ current via the asphingomyelin pathway. Biochem. Biophys. Res. Commun. 344, 189-193. doi: 10.1016/j.bbrc.2006.03.115

Heidt, T., Courties, G., Dutta, P., Sager, H. B., Sebas, M., Iwamoto, Y., et al. (2014). Differential contribution of monocytes to heart macrophages in steadystate and after myocardial infarction. Circ. Res. 115, 284-295. doi: 10.1161/ circresaha.115.303567

Hilgendorf, I., Gerhardt, L. M., Tan, T. C., Winter, C., Holderried, T. A., Chousterman, B. G., et al. (2014). Ly-6Chigh monocytes depend on Nr4al to balance both inflammatory and reparative phases in the infarcted myocardium. Circ. Res. 114, 1611-1622. doi: 10.1161/circresaha.114.303204 
Hirayama, D., Iida, T., and Nakase, H. (2017). The phagocytic function of macrophage-enforcing innate immunity and tissue homeostasis. Int. J. Mol. Sci. 19:E92.

Honold, L., and Nahrendorf, M. (2014). Resident and monocyte-derived macrophages in cardiovascular disease. Circ. Res. 122, 113-127. doi: 10.1161/ circresaha.117.311071

Hu, J., Huang, C. X., Rao, P. P., Zhou, J. P., Wang, X., Tang, L., et al. (2019). Inhibition of microRNA-155 attenuates sympathetic neural remodeling following myocardial infarction via reducing M1 macrophage polarization and inflammatory responses in mice. Eur. J. Pharmacol. 851, 122-132. doi: 10.1016/ j.ejphar.2019.02.001

Hulsmans, M., Clauss, S., Xiao, L., Aguirre, A. D., King, K. R., Hanley, A., et al. (2017). Macrophages facilitate electrical conduction in the heart. Cell 169, 510-522.

Ikeda, U., Ohkawa, F., Seino, Y., Yamamoto, K., Hidaka, Y., Kasahara, T., et al. (1992). Serum interleukin 6 levels become elevated in acute myocardial infarction. J. Mol. Cell Cardiol. 24, 579-584. doi: 10.1016/0022-2828(92)91 042-4

Italiani, P., and Boraschi, D. (2014). From monocytes to M1/M2 macrophages: phenotypical vs. functional differentiation. Front. Immunol. 5:514. doi: 10.3389/ fimmu.2014.00514

Jung, M., Ma, Y., Iyer, R. P., DeLeon-Pennell, K. Y., Yabluchanskiy, A., Garrett, M. R., et al. (2017). IL-10 improves cardiac remodeling after myocardial infarction by stimulating M2 macrophage polarization and fibroblast activation. Basic Res. Cardiol. 112:33.

Kalla, M., Herring, N., and Paterson, D. J. (2016). Cardiac sympatho-vagal balance and ventricular arrhythmia. Auton. Neurosci. 199, 29-37. doi: 10.1016/j.autneu. 2016.08.016

Kao, Y. H., Chen, Y. C., Cheng, C. C., Lee, T. I., Chen, Y. J., and Chen, S. A. (2010). Tumor necrosis factor-alpha decreases sarcoplasmic reticulum Ca2+-ATPase expressions via the promoter methylation in cardiomyocytes. Crit. Care Med. 38, 217-222. doi: 10.1097/ccm.0b013e3181b4a854

Kinugawa, K., Takahashi, T., Kohmoto, O., Yao, A., Aoyagi, T., Momomura, S., et al. (1994). Nitric oxide-mediated effects of interleukin-6 on $[\mathrm{Ca} 2+] \mathrm{i}$ and cell contraction in cultured chick ventricular myocytes. Circ. Res. 75, 285-295. doi: 10.1161/01.res.75.2.285

Klein, R. M., Vester, E. G., Brehm, M. U., Dees, H., Picard, F., Niederacher, D., et al. (2000). Inflammation of the myocardium as an arrhythmia trigger. Zeitschrift Kardiol. 89, 24-35.

Lavine, K. J., Epelman, S., Uchida, K., Weber, K. J., Nichols, C. G., Schilling, J. D., et al. (2014). Distinct macrophage lineages contribute to disparate patterns of cardiac recovery and remodeling in the neonatal and adult heart. Proc. Natl. Acad. Sci. U.S.A. 111, 16029-16034. doi: 10.1073/pnas.1406508111

Lazzerini, P. E., Acampa, M., Capecchi, P. L., Fineschi, I., Selvi, E., Moscadelli, V., et al. (2015a). Antiarrhythmic potential of anticytokine therapy in rheumatoid arthritis: tocilizumab reduces corrected QT interval by controlling systemic inflammation. Arthritis Care Res. 67, 332-339. doi: 10.1002/acr.22455

Lazzerini, P. E., Capecchi, P. L., and Laghi-Pasini, F. (2015b). Long QT syndrome: an emerging role for inflammation and immunity. Front. Cardiovasc. Med. 2:26. doi: $10.3389 /$ fcvm.2015.00026

Lazzerini, P. E., Capecchi, P. L., and Laghi-Pasini, F. (2017a). Systemic inflammation and arrhythmic risk: lessons from rheumatoid arthritis. Eur. Heart J. 38, 1717-1727.

Lazzerini, P. E., Laghi-Pasini, F., Bertolozzi, I., Morozzi, G., Lorenzini, S., Simpatico, A., et al. (2017b). Systemic inflammation as a novel QT-prolonging risk factor in patients with torsades de pointes. Heart 103, 1821-1829. doi: 10.1136/heartjnl-2016-311079

Levick, S. P., Murray, D. B., Janicki, J. S., and Brower, G. L. (2010). Sympathetic nervous system modulation of inflammation and remodeling in the hypertensive heart. Hypertension 55, 270-276. doi: 10.1161/hypertensionaha. 109.142042

Lewek, J., Kaczmarek, K., Cygankiewicz, I., Wranicz, J. K., and Ptaszynski, P. (2014). Inflammation and arrhythmias: potential mechanisms and clinical implications. Expert Rev. Cardiovasc. Ther. 12, 1077-1085. doi: 10.1586/ 14779072.2014 .942286

Li, Y. H., and Rozanski, G. J. (1993). Effects of human recombinant interleukin1 on electrical properties of guinea pig ventricular cells. Cardiovasc. Res. 27, 525-530. doi: $10.1093 / \mathrm{cvr} / 27.3 .525$
Li, Y. Y., Feldman, A. M., Sun, Y., and McTiernan, C. F. (1998). Differential expression of tissue inhibitors of metalloproteinases in the failing human heart. Circulation 98, 1728-1734. doi: 10.1161/01.cir.98.17.1728

Libby, P. (2013). Mechanisms of acute coronary syndromes and their implications for therapy. N. Engl. J. Med. 368, 2004-2013. doi: 10.1056/nejmra1216063

Libby, P., Ridker, P. M., and Maseri, A. (2002). Inflammation and atherosclerosis. Circulation 105, 1135-1143.

Liu, S. J., Zhou, W., and Kennedy, R. H. (1999). Suppression of beta-adrenergic responsiveness of L-type $\mathrm{Ca} 2+$ current by IL-1beta in rat ventricular myocytes. Am. J. Physiol. 276, H141-H148.

London, B., Baker, L. C., Lee, J. S., Shusterman, V., Choi, B. R., Kubota, T., et al. (2003). Calcium-dependent arrhythmias in transgenic mice with heart failure. Am. J. Physiol. Heart Circ. Physiol. 284, H431-H441.

MacEwan, D. J. (2002). TNF receptor subtype signalling: differences and cellular consequences. Cell Signal. 14, 477-492. doi: 10.1016/s0898-6568(01)00262-5

Mantravadi, R., Gabris, B., Liu, T., Choi, B. R., de Groat, W. C., Ng, G. A., et al. (2007). Autonomic nerve stimulation reverses ventricular repolarization sequence in rabbit hearts. Circ. Res. 100, e72-e80.

Marx, S. O., Reiken, S., Hisamatsu, Y., Jayaraman, T., Burkhoff, D., Rosemblit, N., et al. (2000). PKA phosphorylation dissociates FKBP12.6 from the calcium release channel (ryanodine receptor): defective regulation in failing hearts. Cell 101, 365-376. doi: 10.1016/s0092-8674(00)80847-8

Monnerat, G., Alarcón, M. L., Vasconcellos, L. R., Hochman-Mendez, C., Brasil, G., Bassani, R. A., et al. (2016). Macrophage-dependent IL-1 $\beta$ production induces cardiac arrhythmias in diabetic mice. Nat. Commun. 7:13344.

Moore, K. J., Sheedy, F. J., and Fisher, E. A. (2013). Macrophages in atherosclerosis: a dynamic balance. Nat. Rev. Immunol. 13, 709-721. doi: 10.1038/nri3520

Mukherjee, R., Colbath, G. P., Justus, C. D., Bruce, J. A., Allen, C. M., Hewett, K. W., et al. (2010). Spatiotemporal induction of matrix metalloproteinase-9 transcription after discrete myocardial injury. FASEB J. 24, 3819-3828. doi: 10.1096/fj.10-155531

Nahrendorf, M., Swirski, F. K., Aikawa, E., Stangenberg, L., Wurdinger, T., Figueiredo, J. L., et al. (2007). The healing myocardium sequentially mobilizes two monocyte subsets with divergent and complementary functions. J. Exp. Med. 204, 3037-3047. doi: 10.1084/jem.20070885

Ng, G. A., Mantravadi, R., Walker, W. H., Ortin, W. G., Choi, B. R., de Groat, W., et al. (2009). Sympathetic nerve stimulation produces spatial heterogeneities of action potential restitution. Heart Rhythm 6, 696-706. doi: 10.1016/j.hrthm. 2009.01.035

Nguyen, T. P., Qu, Z., and Weiss, J. N. (2014). Cardiac fibrosis and arrhythmogenesis: the road to repair is paved with perils. J. Mol. Cell Cardiol. 70, 83-91. doi: 10.1016/j.yjmcc.2013.10.018

Nian, M., Lee, P., Khaper, N., and Liu, P. (2004). Inflammatory cytokines and postmyocardial infarction remodeling. Circ. Res. 94, 1543-1553. doi: 10.1161/ 01.res.0000130526.20854.fa

Okada, D. R., Smith, J., Derakhshan, A., Gowani, Z., Misra, S., Berger, R. D., et al. (2018). Ventricular arrhythmias in cardiac sarcoidosis. Circulation 138, 1253-1264.

Petkova-Kirova, P. S., Gursoy, E., Mehdi, H., McTiernan, C. F., London, B., and Salama, G. (2006). Electrical remodeling of cardiac myocytes from mice with heart failure due to the overexpression of tumor necrosis factor-alpha. Am. J. Physiol. Heart Circ. Physiol. 290, H2098-H2107.

Pinto, A. R. (2017). A change of heart: a transforming view of cardiac function. NPJ Regen. Med. 2:24.

Pinto, A. R., Godwin, J. W., Chandran, A., Hersey, L., Ilinykh, A., Debuque, R., et al. (2014). Age-related changes in tissue macrophages precede cardiac functional impairment. Aging 6, 399-413. doi: 10.18632/aging.100669

Pinto, J. M., and Boyden, P. A. (1999). Electrical remodeling in ischemia and infarction. Cardiovasc. Res. 42, 284-297. doi: 10.1016/s0008-6363(99)00013-9

Podrid, P. J., Fuchs, T., and Candinas, R. (1990). Role of the sympathetic nervous system in the genesis of ventricular arrhythmia. Circulation 82, I103-I113.

Ridker, P. M., Everett, B. M., Thuren, T., MacFadyen, J. G., Chang, W. H., Ballantyne, C., et al. (2017). Antiinflammatory therapy with canakinumab for atherosclerotic disease. N. Engl. J. Med. 377, 1119-1131.

Robbins, C. S., Chudnovskiy, A., Rauch, P. J., Figueiredo, J. L., Iwamoto, Y., Gorbatov, R., et al. (2012). Extramedullary hematopoiesis generates Ly-6Chigh monocytes that infiltrate atherosclerotic lesions. Circulation 125, 364-374. doi: 10.1161/circulationaha.111.061986 
Rohr, S. (2012). Arrhythmogenic implications of fibroblast-myocyte interactions. Circulation 5, 442-452. doi: 10.1161/circep.110.957647

Rohr, S., Kucera, J. P., Fast, V. G., and Kléber, A. G. (1997). Paradoxical improvement of impulse conduction in cardiac tissue by partial cellular uncoupling. Science 275, 841-844. doi: 10.1126/science.275.53 01.841

Said, M., Becerra, R., Valverde, C. A., Kaetzel, M. A., Dedman, J. R., MundiñaWeilenmann, C., et al. (2011). Calcium-calmodulin dependent protein kinase II (CaMKII): a main signal responsible for early reperfusion arrhythmias. J. Mol. Cell Cardiol. 51, 936-944. doi: 10.1016/j.yjmcc.2011.08.010

Sawaya, S. E., Rajawat, Y. S., Rami, T. G., Szalai, G., Price, R. L., Sivasubramanian, N., et al. (2007). Downregulation of connexin 40 and increased prevalence of atrial arrhythmias in transgenic mice with cardiac-restricted overexpression of tumor necrosis factor. Am. J. Physiol. Heart Circ. Physiol. 292, H1561-H1567.

Saxena, A., Chen, W., Su, Y., Rai, V., Uche, O. U., Li, N., et al. (2013). IL-1 induces proinflammatory leukocyte infiltration and regulates fibroblast phenotype in the infarcted myocardium. J. Immunol. 191, 4838-4848. doi: 10.4049/ jimmunol.1300725

Shen, M. J., and Zipes, D. P. (2014). Role of the autonomic nervous system in modulating cardiac arrhythmias. Circ. Res. 114, 1004-1021. doi: 10.1161/ circresaha.113.302549

Shimodaira, T., Matsuda, K., Uchibori, T., Sugano, M., Uehara, T., and Honda, T. (2018). Upregulation of osteopontin expression via the interaction of macrophages and fibroblasts under IL-1b stimulation. Cytokine 110, 63-69. doi: $10.1016 /$ j.cyto.2018.04.025

Sivakumar, P., Gupta, S., Sarkar, S., and Sen, S. (2008). Upregulation of lysyl oxidase and MMPs during cardiac remodeling in human dilated cardiomyopathy. Mol. Cell. Biochem. 307, 159-167. doi: 10.1007/s11010-007-9595-2

Streitner, F., Kuschyk, J., Veltmann, C., Brueckmann, M., Streitner, I., Brade, J., et al. (2007). Prospective study of interleukin-6 and the risk of malignant ventricular tachyarrhythmia in ICD-recipients-a pilot study. Cytokine 40, 3034. doi: $10.1016 /$ j.cyto.2007.07.187

Streitner, F., Kuschyk, J., Veltmann, C., Ratay, D., Schoene, N., Streitner, I., et al. (2009). Role of proinflammatory markers and NT-proBNP in patients with an implantable cardioverter-defibrillator and an electrical storm. Cytokine 47, 166-172. doi: 10.1016/j.cyto.2009.06.003

Su, Q., Lv, X., Sun, Y., Ye, Z., Kong, B., and Qin, Z. (2018). Role of TLR4/MyD88/NF- $\mathrm{KB}$ signaling pathway in coronary microembolizationinduced myocardial injury prevented and treated with nicorandil. Biomed. Pharmacother. 106, 776-784. doi: 10.1016/j.biopha.2018.07.014

Thomas, C. V., Coker, M. L., Zellner, J. L., Handy, J. R., Crumbley, A. J. III, and Spinale, F. G. (1998). Increased matrix metalloproteinase activity and selective upregulation in LV myocardium from patients with end-stage dilated cardiomyopathy. Circulation 97, 1708-1715. doi: 10.1161/01.cir.97.1 7.1708

Tomaselli, G. F., and Zipes, D. P. (2004). What causes sudden death in heart failure? Circ. Res. 95, 754-763. doi: 10.1161/01.res.0000145047.14691.db

Turkdogan, K. A., Zorlu, A., Guven, F. M., Ekinozu, I., Eryigit, U., and Yilmaz, M. B. (2017). Usefulness of admission matrix metalloproteinase 9 as a predictor of early mortality after cardiopulmonary resuscitation in cardiac arrest patients. Am. J. Emerg. Med. 30, 1804-1809. doi: 10.1016/j.ajem.2012.02.017

Udagawa, N. (2003). The mechanism of osteoclast differentiation from macrophages: possible roles of $\mathrm{T}$ lymphocytes in osteoclastogenesis. J. Bone Miner. Metab. 21, 337-343. doi: 10.1007/s00774-003-0439-1

Ukena, C., Mahfoud, F., Kindermann, I., Kandolf, R., Kindermann, M., and Böhm, M. (2011). Prognostic electrocardiographic parameters in patients with suspected myocarditis. Eur. J. Heart Fail. 13, 398-405. doi: 10.1093/eurjhf/ hfq229

van der Laan, A. M., Ter Horst, E. N., Delewi, R., Begieneman, M. P., Krijnen, P. A., Hirsch, A., et al. (2014). Monocyte subset accumulation in the human heart following acute myocardial infarction and the role of the spleen as monocyte reservoir. Eur. Heart J. 35, 376-385. doi: 10.1093/eurheartj/eht331
Vaseghi, M., Lux, R. L., Mahajan, A., and Shivkumar, K. (2012). Sympathetic stimulation increases dispersion of repolarization in humans with myocardial infarction. Am. J. Physiol. Heart Circ. Physiol. 302, H1838-H1846.

Vonderlin, N., Siebermair, J., Kaya, E., Köhler, M., Rassaf, T., and Wakili, R. (2019). Critical inflammatory mechanisms underlying arrhythmias. Herz 44, 121-129. doi: 10.1007/s00059-019-4788-5

Wakili, R., Voigt, N., Kääb, S., Dobrev, D., and Nattel, S. (2011). Recent advances in the molecular pathophysiology of atrial fibrillation. J. Clin. Invest. 121, 2955-2968. doi: 10.1172/jci46315

Wang, M., Li, S., Zhou, X., Huang, B., Zhou, L., Li, X., et al. (2017). Increased inflammation promotes ventricular arrhythmia through aggravating left stellate ganglion remodeling in a canine ischemia model. Int. J. Cardiol. 248, 286-293. doi: 10.1016/j.ijcard.2017.08.011

Wang, Y., Xuan, Y. L., Hu, H. S., Li, X. L., Xue, M., Cheng, W. J., et al. (2012). Risk of ventricular arrhythmias after myocardial infarction with diabetes associated with sympathetic neural remodeling in rabbits. Cardiology 121, 1-9. doi: 10. $1159 / 000336148$

Wehrens, X. H., Lehnart, S. E., Reiken, S. R., Deng, S. X., Vest, J. A., Cervantes, D., et al. (2004). Protection from cardiac arrhythmia through ryanodine receptorstabilizing protein calstabin2. Science 304, 292-296. doi: 10.1126/science. 1094301

Weng, C. H., Chung, F. P., Chen, Y. C., Lin, S. F., Huang, P. H., Kuo, T. B., et al. (2016). Pleiotropic effects of myocardial MMP-9 inhibition to prevent ventricular arrhythmia. Sci. Rep. 6:38894.

Wernli, G., Hasan, W., Bhattacherjee, A., van Rooijen, N., and Smith, P. G. (2009). Macrophage depletion suppresses sympathetic hyperinnervation following myocardial infarction. Basic Res. Cardiol. 104, 681-693. doi: 10.1007/s00395009-0033-3

Yang, N., Cheng, W., Hu, H., Xue, M., Li, X., Wang, Y., et al. (2016). Atorvastatin attenuates sympathetic hyperinnervation together with the augmentation of M2 macrophages in rats postmyocardial infarction. Cardiovasc. Therap. 34, 234-244. doi: 10.1111/1755-5922.12193

Yang, S., Zheng, R., Hu, S., Ma, Y., Choudhry, M. A., Messina, J. L., et al. (2004). Mechanism of cardiac depression after trauma-hemorrhage: increased cardiomyocyte IL- 6 and effect of sex steroids on IL-6 regulation and cardiac function. Am. J. Physiol. Heart Circ. Physiol. 287, H2183-H2191.

Yap, J., Cabrera-Fuentes, H. A., Irei, J., Hausenloy, D. J., and Boisvert, W. A. (2019). Role of macrophages in cardioprotection. Int. J. Mol. Sci. 20:2474. doi: 10.3390/ijms 20102474

Yin, J., Hu, H., Li, X., Xue, M., Cheng, W., Wang, Y., et al. (2016). Inhibition of Notch signaling pathway attenuates sympathetic hyperinnervation together with the augmentation of M2 macrophages in rats post-myocardial infarction. Am. J. Physiol. Cell Physiol. 310, C41-C53.

Yu, X., Kennedy, R. H., and Liu, S. J. (2003). JAK2/STAT3, not ERK1/2, mediates interleukin-6-induced activation of inducible nitricoxide synthase and decrease in contractility of adult ventricular myocytes. J. Biol. Chem. 278, 16304-16309. doi: 10.1074/jbc.m212 321200

Zipes, D. P., and Rubart, M. (2006). Neural modulation of cardiac arrhythmias and sudden cardiac death. Heart Rhythm 3, 108-113. doi: 10.1016/j.hrthm.2005.09. 021

Conflict of Interest: The authors declare that the research was conducted in the absence of any commercial or financial relationships that could be construed as a potential conflict of interest.

Copyright (c) 2020 Chen, Li, Wang, Yu, Tang and Zhou. This is an open-access article distributed under the terms of the Creative Commons Attribution License (CC BY). The use, distribution or reproduction in other forums is permitted, provided the original author(s) and the copyright owner(s) are credited and that the original publication in this journal is cited, in accordance with accepted academic practice. No use, distribution or reproduction is permitted which does not comply with these terms. 